\title{
Time-Domain Simulations of a 31-Antenna Array for Breast Cancer Imaging
}

\author{
Maciej Klemm \\ Department of Electrical and Electronic Engineering \\ University of Bristol, United Kingdom \\ m.klemm@bristol.ac.uk
}

Dirk Baumann

Laboratory for Electromagnetic Fields and Microwave Electronics

ETH Zurich, Switzerland

dbaumann@ifh.ee.ethz.ch

\author{
Christophe Fumeaux \\ School of Electrical and Electronic Engineering \\ University of Adelaide, Australia \\ cfumeaux@eleceng.adelaide.edu.au \\ Ian J. Craddock \\ Department of Electrical and Electronic Engineering \\ University of Bristol, United Kingdom \\ i.j.craddock@bristol.ac.uk
}

\begin{abstract}
In this paper we discuss challenges related with timedomain simulations of a complete microwave radar imaging system for breast cancer detection. Two different numerical methods are considered to address this demanding electromagnetic problem featuring 31 ultra-wideband antennas. The first method is the Finite Integration Technique (FIT) applied in a regular grid and implemented in a commercial solver, whereas the second method is an in-house developed FiniteVolume Time-Domain (FVTD) code applied in a tetrahedral mesh. Our work focuses on the fundamental differences between the two approaches for the comprehensive full-wave modeling of the considered problem. The emphasis of the comparison is placed on the computational cost, which reveals the strengths and limitations of both methods for the problem considered.
\end{abstract}

Keywords- microwave imaging, ultra-wideband antennas, antenna array, computational electromagnetics

\section{INTRODUCTION}

X-ray mammography is currently the most common technique used in breast cancer screening. It employs ionizing radiation, requires uncomfortable compression of the breast during the examination and is of limited value for younger women. These limitations of X-ray mammography have resulted in research into alternative methods for imaging breast cancer. Microwave imaging is one of the more promising candidates and has attracted the interest of a number of research groups around the world. An excellent review of this topic can be found in [1]. As presented there, currently there are two main streams in microwave breast imaging: a) microwave tomography, b) radar-based imaging. Both approaches rely on the difference in the electrical properties of normal and malignant breast tissues.

In this paper we focus on numerical modeling of the radar imaging system developed at Bristol University and recently reported in [2-3]. In radar-based imaging, the goal is to create a

M. Klemm acknowledges the support of the UK's Engineering and Physical Sciences Research Council (EPSRC). C. Fumeaux acknowledges the support of the Australian Research Council (ARC) Future Fellowship funding scheme (project number FT100100585). map of microwave scattering, arising from the contrast in dielectric properties within the breast. The accurate numerical modeling of the full system, including a breast phantom, is a crucial step to support further development of the technique.

Considering the evolution of microwave imaging systems (radar or tomographic) towards systems with multiple compact directive antennas, the full-wave simulation of such a system including fine details becomes a very challenging task. Until recently numerical models of antenna arrays (the most important part of the imaging system) were limited to simple sources or dipole antennas, as presented for the radar [4] and tomographic [5] imaging system. However, as presented in [6] and [4] by choosing more complex antenna with better performance as well as adding more elements into the array, one can achieve a significant improvement in imaging performance. We therefore believe that further development of microwave imaging systems will require numerical electromagnetic (EM) modeling of the complete imaging system with accurate models of complex antenna designs, as well as the breast phantom composed of realistic biological tissue. In this paper we will present some first attempts towards this goal.

Time-domain methods appear attractive to simulate radar imaging systems relying on pulsed UWB operation. In the present investigation, we compare the application of two timedomain methods to model this problem in an acceptable time. The two numerical techniques are the Finite Integration Technique (FIT) and the Finite-Volume Time-Domain (FVTD) method, which are representative of different approaches towards tackling such a complex numerical problem, which is huge in terms of number of variables. The paper first briefly introduces the features of the two methods relevant for the current problem. It then considers the simulation in increasing levels of complexity: From a single antenna oriented along principal axis, to a squinted antenna, and towards the 31 antenna-system simulated with breast phantom. The concluding discussion provides reflections on the strengths and limitations of the two approaches. 


\section{Time-Domain Simulation Methods}

The presented investigation considers only full-wave timedomain numerical methods, as they appear well suited for modeling transient/UWB operation. The methods being well documented in the literature, only the most salient characteristics and their differences relevant for the present study are briefly summarized here.

\section{A. FIT}

The FIT [7] is a commonly applied time-domain method for electromagnetic simulations. It is characterized by second-order accuracy in space and time at a minimal computational cost per rectangular cell. Parallelization is straightforward. The commercial code used in the present study is $\mathrm{CST}^{\circledR}$ Microwave Studio. Most features and conclusions for FIT drawn in this paper would also be true for the Finite-Difference Time-Domain (FDTD) [8] method, as both methods results in similar update equations when implemented in a Cartesian grid.

\section{B. FVTD}

The FVTD method has been introduced at the end of the 1990's [9], [10]. It never became a mainstream method but is representative of time-domain methods applied in unstructured meshes. Other approaches with similar features are the tetrahedral Transmission Line Modeling (TLM) [11] the FiniteElement Time-Domain (FETD) [12] and the Discontinuous Galerkin Time-Domain (DG-TD) [13] methods. The FVTD method has found niche applications because of its geometrical flexibility, e.g. for scattering and radiation problems with strong inhomogeneities and small details embedded in larger structures, e.g. [14], [15]. The in-house implementation of the FVTD technique used in this study is based on a cell-centered scheme with upwind fluxes [16]. The efficiency of the solver is increased through a local time-stepping scheme [17] which combines the inhomogeneous spatial tetrahedral discretization with an inhomogeneous temporal discretization. Dispersive materials are handled as described in [18] and the computational domain can be truncated using spherical or conformal perfectlymatched absorbers [19]. The 31 ports are defined using the technique introduced in [20].

\section{Wide-SLOt CAVITY-ENClosed UWB ANTENNA}

\section{A. Geometry}

The antenna array of the radar imaging system is comprised of 31 wide-slot UWB antennas enclosed in the cavity (shown in Fig. 1). A detailed design description of the single element antenna is reported in [6]. The main advantages of this design are a stable radiation pattern across the frequency band of interest $(4-10 \mathrm{GHz})$ in the operating oil medium with a permittivity of $\varepsilon_{r} \approx 9$, as well as extremely high fidelity (above $95 \%$ ) of radiated pulses for radiation angles even up to $60^{\circ}$ from bore-sight.

\section{B. Comparison of FIT and FVTD Models}

The discretization of the wide-slot antenna backed by an absorber-loaded cavity is shown in Fig. 2 for both numerical methods, i.e. a tetrahedral mesh for FVTD and Cartesian grid for FIT.
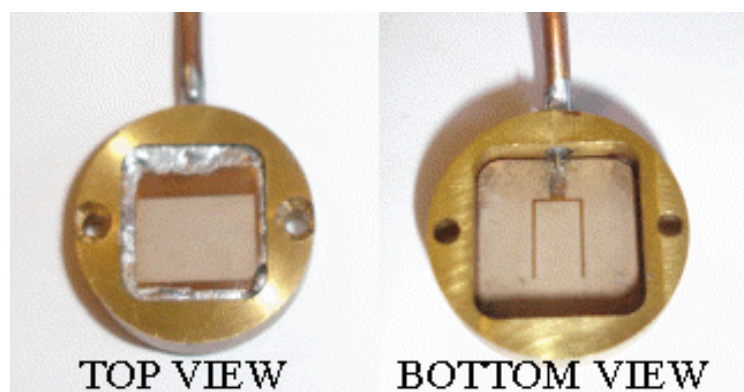

BOTTOM VIEW

Figure 1. Photo of the wide-slot UWB antenna used in microwave-radar imaging system. Antenna is mounted in the brass, which is attached to the cavity (not shown herein). The cavity has an inner dimension of $14 \times 14 \mathrm{~mm}$.
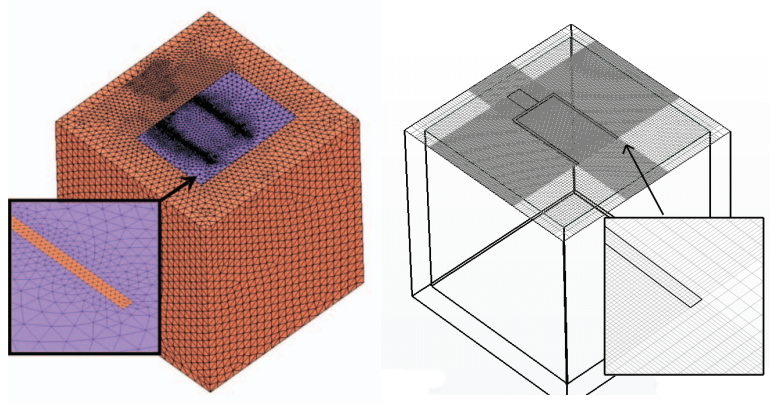

Figure 2. Discretization of the antenna Left: Triangular surface mesh used in the FVTD simulation. The tetrahedral volume mesh is constructed on the basis of this surface mesh. Refinement is visible on the feeding fork (inset). Right: FIT grid showing the non-uniform mesh around the fork.

From the point of view of numerical simulation, a difficult feature of this antenna is the resolution of the thin fork feeding section, which exhibits a width of $0.2 \mathrm{~mm}$. This dimension corresponds to a small fraction of the wavelength $(<\lambda / 50)$ in the operation medium. In order to correctly approximate the transverse current distribution on this fork, a discretization comprising at least 3 transverse sample points is required. This is addressed differently in the two considered methods. The FIT requires a very fine mesh extending over the full transverse side of the computational domain to resolve this feature, whereas the tetrahedral mesh can accommodate different scales of details by adapting the cell size to the required spatial resolution needed locally. This second option might appear more elegant and efficient at first view, however it has to be balanced by the fact that the treatment of a FIT cell is computationally cheaper by approximately an order of magnitude, than the treatment of a tetrahedral mesh, both in terms of CPU time and memory.

Another very important aspect regards the orientation of the antenna in the coordinate system. Considering that all the surfaces are orientated orthogonally to each other, the simulation of a single antenna aligned along the principal xyz coordinate axes can be performed very efficiently in a Cartesian grid, i.e. with FIT. When however the antenna is tilted at an arbitrary angle in the coordinate system, as is required for the simulation of the conformal array, the resolution of the slanted surfaces increases the computational efforts drastically. In contrast, the simulation in an unstructured mesh might appear costly when performed for the antenna aligned in the coordinate 
system, but is totally independent on the orientation of the antenna.

The orientation of the antenna is also related to a third relevant aspect, which regards the modeling of the antenna excitation. As shown in Fig. 1 the antenna is fed directly from the coaxial cable soldered to the antenna's microstrip feedline. The same coaxial feed can be readily implemented in both numerical models. However, when the antenna is not aligned with the Cartesian grid, the standard coaxial excitation cannot be used in the FIT model and need to be replaced by a voltage gap excitation (with $50 \Omega$ resistor). Moreover, for different antenna in the array, a voltage gap excitation will vary due to differences in mesh size. This can have quite unpredictable effects on the modeled matching of the antenna.

The measured input reflection coefficient for the realized antennas is presented in Fig. 3, together with data from three corresponding simulated models. A good agreement is achieved between the measurement and simulations when the coaxial excitation is used in FVTD and FIT. The fine structure of the resonances is not perfectly retrieved because of the actual differences between the simulated and realized device, but both methods correctly predict the operation bandwidth of the antenna. However, a significantly different result is obtained for the FIT model with the voltage gap feed, especially at higher frequencies. This might have a noticeable effect on the full system simulation where the elements are tilted to realize a conformal arrangement.

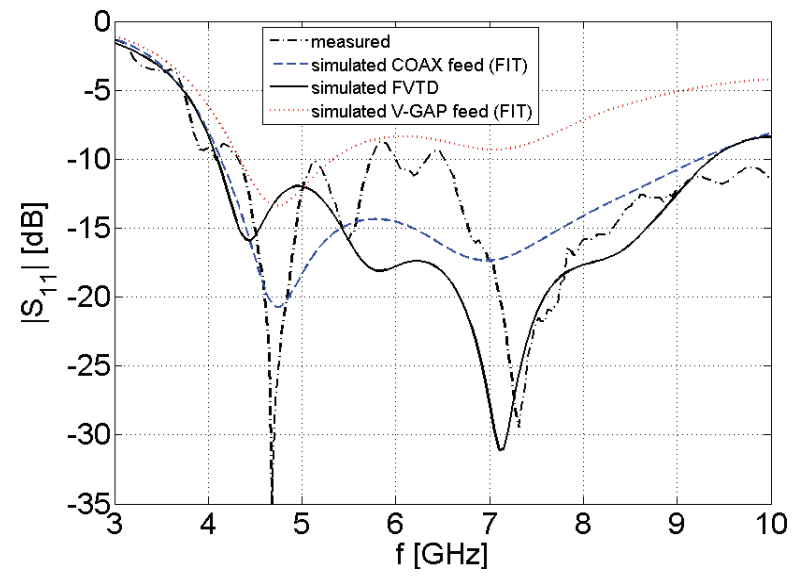

Figure 3. Comparison of simulated and measured reflection coefficient for a single element UWB wide-slot antenna.

Indications of the computational cost associated with the simulations using the different methods are given in Table I. It is emphasized that these results are not intended as a fair and absolute comparison, since the results were computed on different machines, but these data illustrate the description of the different challenges associated with the different methods. The FVTD method has a relatively high cost per cell, but the unstructured mesh allows saving on the number of discretizing cells. It is additionally independent on the antenna orientation. The FIT can handle very efficiently large number of cells, but the detailed geometry of this antenna, especially when tilted is putting strains on the computer resources.
TABLE I. COMPUtational COSTS For A Single ElEMENT

\begin{tabular}{|c|c|c|}
\hline Model & FVTD $^{(2)}$ & FIT \\
\hline \multicolumn{3}{|l|}{ Single antenna } \\
\hline \# mesh cells & $750 \mathrm{k}$ & $10.3 \mathrm{M}^{(3)}$ \\
\hline Memory & $0.6 \mathrm{~GB}$ & $0.9 \mathrm{~GB}$ \\
\hline CPU time ${ }^{(1)}$ & $4.8 \mathrm{~h}$ & $2 \mathrm{~h}$ \\
\hline \multicolumn{3}{|l|}{ Tilted antenna } \\
\hline \# mesh cells & $750 \mathrm{k}$ & $24.5 \mathrm{M}^{(3)}$ \\
\hline Memory & $0.6 \mathrm{~GB}$ & $2 \mathrm{~GB}$ \\
\hline CPU time ${ }^{(1)}$ & $4.8 \mathrm{~h}$ & $7 \mathrm{~h}$ \\
\hline
\end{tabular}

${ }^{\text {(1) }}$ FVTD computations were performed on a single core of an Intel Xeon E5620@2.40 GHz. FIT computations were performed on two quad core Intel Xeon E5405@2.0GHz ( 8 cores in total used).

${ }^{(2)}$ In spherical domain with radius $25 \mathrm{~mm}$, for $2 \mathrm{~ns}$ simulation.

(3) $50 \mathrm{~mm}$ distance from the antenna to the UPML boundary in all directions.

\section{Simulation OF THE CONFORMAL ARRAY}

\section{A. Geometry}

An antenna array (Fig. 4) is formed around the lower part of a $85 \mathrm{~mm}$ radius sphere. To provide the best radiation coverage of a breast, all antennas were positioned to point towards a center of curvature. A plastic shell with openings for the antennas has been manufactured to assure the best possible accuracy of positioning antennas. In normal operation conditions all antennas are immersed in a matching liquid with dielectric constant of around 9 and losses $4 \mathrm{~dB} / \mathrm{cm}$ (at $10 \mathrm{GHz}$ ).
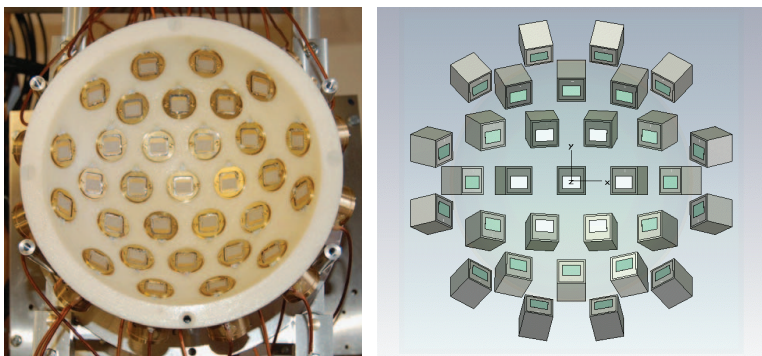

Figure 4. 31-element antenna array designed for microwave breast cancer detection. Physical (left) and numerical (right) models shown.

\section{B. Comparison of FIT and FVTD Models}

Fig. 5(a) presents a partial numerical model, which only considers transmission between two UWB wide-slot antennas through a dispersive breast phantom. The antennas are selected from two opposite ends of the array as shown in Fig. 4. The breast model includes a $2 \mathrm{~mm}$ thick skin layer and a homogeneous fatty tissue forming the interior of the breast. Both tissues are dispersive with Debye model parameters as follows: skin $\left(\varepsilon_{s}=37, \varepsilon_{\text {inf }}=4, \tau=7.2 \mathrm{ps}\right)$, fatty breast tissue $\left(\varepsilon_{\mathrm{s}}=10, \quad \varepsilon_{\text {inf }}=7, \tau=7 \mathrm{ps}\right)$. Results of the simulations are presented in Fig. 5(b). A good agreement is achieved between two numerical methods. An effect of the lossy dispersive tissues is clearly visible, limiting the effective bandwidth of the imaging system. This partial simulation is used as validation for full simulations of the complete array comprising all 31 antennas. 


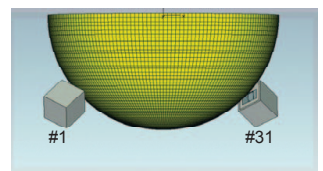

(a)

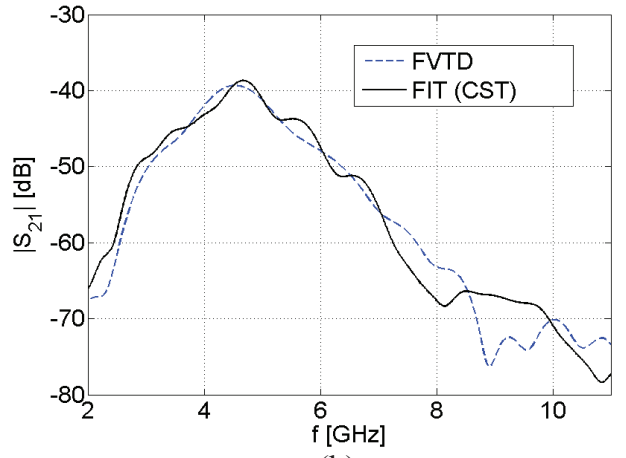

(b)

Figure 5. Transmission between two UWB antennas from the array through a dispersive breast phantom. (a) Numerial model of antennas and breast, (b) Simulated transmission coefficient.

Indications of the computational cost for the modeling of the array antenna including phantom are given in Table II. Again, the listed results are not to be interpreted as fair and absolute comparison, but are meant to illustrate the different constraints on the two selected computational methods. The data demonstrate that main challenge for the FVTD method is the large dispersive phantom, whereas the main hurdle for the FIT method is rather the details of the 31 tilted antennas.

\section{CONCLUSION}

The paper compared the capabilities and limitations of two time-domain electromagnetic simulation tools. Whereas FIT is applied in a structured mesh, the FVTD method employs an unstructured mesh. Using the challenging example of a microwave breast-cancer imaging system, the computational efficiency of the different mesh topologies was investigated. It was shown that both computational approaches appeared viable for the problem at hand. However, both methods showed different strengths, which could be exploited advantageously. Further investigations including the full array consisting of 31 antennas are currently performed.

TABLE II. COMPUTATIONAL COSTS FOR THE ARRRAY WITH PHANTOM

\begin{tabular}{|c|c|c|}
\hline Model & FVTD & FIT \\
\hline 2-antenna model & \multicolumn{2}{|}{} \\
\hline \# mesh cells & $11 \mathrm{M}$ & $118 \mathrm{M}$ \\
\hline Memory & $17 \mathrm{~GB}$ & $10.2 \mathrm{~GB}$ \\
\hline CPU time (for 5 ns) ${ }^{(1)}$ & $180 \mathrm{~h}$ & $107 \mathrm{~h}$ \\
\hline 31-antenna model & \multicolumn{1}{|}{} \\
\hline \# mesh cells & $22 \mathrm{M}$ & $>800 \mathrm{M}\left(450 \mathrm{M}^{(2)}\right)$ \\
\hline Memory & $25 \mathrm{~GB}$ & $>48 \mathrm{~GB}\left(40 \mathrm{~GB}^{(2)}\right)$ \\
\hline CPU time (for 5 ns) ${ }^{(1)}$ & $404 \mathrm{~h}$ & $\mathrm{~N} / \mathrm{A}\left(504 \mathrm{~h}^{(2)}\right)$ \\
\hline
\end{tabular}

${ }^{(1)}$ FVTD computations were performed on a single core of a Intel Xeon E5620

(a) $2.40 \mathrm{GHz}$. FIT computations were performed on two quad core Intel Xeon E5405@2.0GHz (8 cores used in total).

${ }^{(2)}$ FIT computations achieved by dividing the problem into three parts.

\section{REFERENCES}

[1] E.C. Fear, P.M. Meaney, M.A. Stuchly, "Microwaves for breast cancer detection?", IEEE Potentials, vol. 22, no. 1, pp. 12-18, Feb-Mar 2003.

[2] M. Klemm, J.A. Leendertz, D. Gibbins, I.J. Craddock, A. Preece, R. Benjamin, "Microwave Radar-Based Breast Cancer Detection: Imaging in Inhomogeneous Breast Phantoms," IEEE Antennas Wireless Propagat. Lett., vol.8, no., pp.1349-1352, 2009.

[3] M. Klemm, I. J. Craddock, A. Preece, J. Leendertz, and R. Benjamin, "Microwave Radar-based Differential Breast Cancer Imaging: Part 1 Imaging in Homogeneous Breast Phantoms and Low Contrast Scenarios", IEEE Trans. Antennas Propagat. vol. 58, no.7, pp. 2337-2344, June 2010.

[4] M. Klemm, et al, "Towards contrast enhanced breast imaging using ultrawideband microwave radar system," IEEE Radio and Wireless Symposium (RWS), pp.516-519, 10-14 Jan. 2010.

[5] J.D. Shea, S.C. Hagness, B.D. Van Veen, "Hardware acceleration of FDTD computations for 3-D microwave breast tomography," IEEE Antennas and Propagation Society International Symposium 2009, APSURSI '09, pp.1-4, 1-5 June 2009.

[6] D. Gibbins, M. Klemm, I.J. Craddock, J.A. Leendertz, A. Preece, R. Benjamin, "A Comparison of a Wide-Slot and a Stacked Patch Antenna for the Purpose of Breast Cancer Detection," IEEE Trans. Antennas Propagat., vol.58, no.3, pp. 665-674, March 2010.

[7] T. Weiland, "A Discretization Method for the Solution of Maxwell's Equations for Six-Component Fields" Electronics and Communications AEUE, vol. 31, no. 3, pp. 116-120, 1977.

[8] A. Taflove and S. C. Hagness, Computational Electrodynamics: The Finite- Difference Time-Domain Method, 3rd ed. Norwood, MA: Artech House, 2005.

[9] N. K. Madsen and R. W. Ziolkowski, "A three-dimensional modified finite volume technique for Maxwell's equations," Electromagn., vol. 10, pp. 147-161, 1990.

[10] V. Shankar, A. H. Mohammadian, and W.F. Hall, "A time-domain, finitevolume treatment for the Maxwell equations," Electromagn., vol. 10, pp. $127-145,1990$

[11] P.D. Sewell, T.M. Benson, C. Christopoulos, D.W.P. Thomas, A. Vukovic, and J.G. Wykes, "Implicit element clustering for tetrahedral transmission-line modeling (TLM)", IEEE Transactions on Microwave Theory Tech., vol. 57, no. 8, pp. 2005-2014, August 2009.

[12] J. F. Lee, R. Lee, and A. C. Cangellaris, "Time-domain finite element methods," IEEE Trans. Antennas Propagat., vol. 45, pp. 430-442, 1997.

[13] J. S. Hesthaven and T. Warburton, Nodal Discontinuous Galerkin Methods: Analysis, Algorithms, and Applications. Springer-Verlag, Berlin, Germany, 2008.

[14] C. Fumeaux, D. Baumann, and R. Vahldieck, "Finite-Volume TimeDomain analysis of a cavity-backed Archimedean spiral antenna", IEEE Trans. Antennas and Propagat., vol. 54, no. 3, pp. 844-851, March 2006.

[15] D.K. Firsov and J. LoVetri, "FVTD - Integral Equation Hybrid for Maxwell's Equations", Int. J. Numer. Model.vol. 21, pp. 29-42, 2007.

[16] P. Bonnet, X. Ferrieres, B. Michielsen, P. Klotz, and J. Roumiguieres, Chap. 9., Time Domain Electromagnetics, Academic Press, 1999.

[17] C. Fumeaux, D. Baumann, P. Leuchtmann, and R. Vahldieck, "A generalized local time-step scheme for efficient FVTD simulations in strongly inhomogeneous meshes", IEEE Trans. Microwave Theory Tech., vol. 52, no. 3, pp. 1067-1076, March 2004.

[18] D. Baumann, C. Fumeaux, C. Hafner, and E.P. Li, "A modula implementation of dispersive materials for time-domain simulations with application to gold nanospheres at optical frequencies", Optics Express vol 17, no. 17, pp. 15186-15200, August 2009.

[19] C. Fumeaux, K. Sankaran, and R. Vahldieck, "Spherical perfectly matched absorber for finite-volume 3-D domain truncation", IEEE Trans. Microwave Theory Tech.. vol. 55, no. 12, pp. 2773-2781, December 2007.

[20] D. Baumann, C. Fumeaux, and R. Vahldieck, "Field-based scatteringmatrix extraction scheme for the FVTD method exploiting a flux-splitting algorithm", IEEE Trans. Microwave Theory Tech. vol. -53, no. 11, pp. 3595-3605, November 2005. 